\title{
Afectos, Emociones Y Sentimientos De Los Estudiantes Panameños Hacia La Matemática Y Su Aprendizaje
}

\author{
Dra. Analinnette Lebrija, \\ Decana de Investigación \\ Universidad Especializada de las Américas. ( Panamá) \\ Dr. Jaime Gutiérrez, \\ Profesor titular, Universidad de Panamá (Panamá) \\ Dra. Mayra Trejos Alvarado \\ Profesor titular, Universidad de Panamá (Panamá)
}

doi: 10.19044/esj.2016.v13n2p281 URL:http://dx.doi.org/10.19044/esj.2016.v13n2p281

\begin{abstract}
We report partial results of an action-dynamic research, of descriptive type, conducted by a learning community that organizes and structures the educational process according to events that occur during a school year; it is based on the constructivist method and the solution of real problems, primarily. It is centered on beliefs about affect, emotions and feelings of Panamanian seventh grade students towards the learning of Mathematics, related to Math Science, Math Class, the Learning of Math and Learning Strategies. The learning community has considered the influence that these factors have on the process of knowledge construction of vital importance. Some important results are as follows. In relation to Science and the motivation towards it, the intervention changed the percentages, for example, from $70.8 \%$ to $100.0 \%$, as far as the taste for the class that receives $72.4 \%$ were interested in studying Mathematics, $93.5 \%$ of the students said at the end of the school year that all students can learn mathematics. Initially, 89.3\% believed to know learning strategies, the percentage in this criterion decreased, this was due to the researchers' intervention better understood their methodology.

From these partial analyses we can observe evidence for the possibilities and challenges of change in the teaching and learning of Math. These results are part of a larger research whose general objective is to design, develop and evaluate a program for teaching and learning Mathematics, based on the modification of negative learning believes from students, and a model for
\end{abstract}


applying daily-life problem-solving strategies, structured on the pre-media curriculum of Panama, that are not part of this article.

Keywords: Beliefs, Feelings, Emotions, Feelings, Learning of Mathematics

\section{Resumen}

En este artículo se reportan resultados parciales de una investigación acción-dinámica, de tipo descriptivo, llevada a cabo por una comunidad de aprendizaje que organiza y estructura el proceso educativo según eventos que se producen durante el año escolar; se fundamenta en el método constructivista y en la solución de problemas reales, primordialmente. Este escrito se centra en las creencias sobre afectos, emociones y sentimientos de los estudiantes panameños de séptimo nivel hacia el aprendizaje de la Matemática relativos a la Ciencia Matemática, clase de Matemática, el aprendizaje de la Matemática y las estrategias de aprendizaje; la comunidad de aprendizaje consideró de vital importancia la influencia de estos factores en el proceso de construcción del conocimiento. Algunos resultados importantes son los siguientes. En relación a la Ciencia y la motivación hacia ella, la intervención hizo cambiar los porcentajes, por ejemplo, de 70,8 \% a $100,0 \%$, en cuanto al gusto hacia la clase que reciben el $72,4 \%$ estuvieron interesados en estudiar Matemática, el 93,5 \% de los educandos opinaron al finalizar el año escolar que todos los estudiantes pueden aprender Matemática. Inicialmente, el 89.3\% creían saber estrategias de aprendizaje, el porcentaje en este criterio descendió, esto se debió a que con la intervención de los investigadores comprendieron mejor su metodología. A partir de estos análisis parciales se pueden observar evidencias de las posibilidades y dificultades del cambio en la enseñanza y aprendizaje de la Matemática. Estos resultados forman parte de una investigación más amplia cuyo objetivo general es diseñar, desarrollar y evaluar un programa de enseñanza y aprendizaje de la Matemática basado en la modificación de creencias negativas de aprendizaje de los alumnos y un modelo de aplicación de estrategias de solución de problemas cotidianos estructurado sobre el currículo de premedia de Panamá, que no forman parte de este artículo.

Palabras clave: Creencias, Afectos, Emociones, sentimientos, Estrategias de aprendizaje

\section{Introducción}

Los continuos resultados negativos y críticas acerca de los deficientes conocimientos matemáticos de los estudiantes de la enseñanza preuniversitaria nos conducen a propuestas en la enseñanza de la Matemática que posibiliten un cambio en su aprendizaje. Algunos datos en los que se 
basan estas afirmaciones: más del $80 \%$ de los alumnos de noveno y duodécimo grado y entre el $50 \%$ y $60 \%$ de los de tercer y sexto grado mostraron un nivel de rendimiento "deficiente", en una escala de excelente, regular y deficiente, según el informe del Programa de Promoción de la Reforma Educativa en América Latina y el Caribe (PREAL, 2007). En PISA 2009 (OECD, 2010), Panamá ocupa el puesto 62 de 65 países, mientras que en Matemática consigue una puntuación de 360, significativamente por debajo del promedio de la OCDE que es 496. En el promedio de Matemática en $3^{\circ}$ grado fue 664 y en $6^{\text {a }}$ grado fue de 644 de 700, ubicando a Panamá con una media significativamente inferior al promedio de los países. Después de esta fecha las autoridades panameñas deciden sacar al País de estas evaluaciones, por lo que no se tienen estadísticas actuales.

En la Universidad de Panamá, en el año 2004, se forma un grupo de profesores universitarios para investigar causas que conduzcan a plantear soluciones a la problemática. Los resultados obtenidos por el grupo investigador se exponen en estudios publicados en Lebrija, Flores, Trejos, 2006, 2010; Lebrija, Trejos, 2005; Lebrija, 2006, 2010, Lebrija 2012, Sáenz, Lebrija 2014. Con el abordaje constructivista, se fundamenta, en el 2015, una investigación cuyo objetivo es: diseñar, desarrollar y evaluar un programa de enseñanza y aprendizaje de la Matemática basado en la modificación de creencias negativas de aprendizaje de los alumnos y un modelo de aplicación de la estrategia para la solución de problemas estructurado sobre el currículo de pre-media de la República de Panamá. La estrategia de solución de problemas sustituye la aplicación mecánica de un algoritmo dentro de un ejercicio. El conocimiento se construye utilizando textos del Centro de Investigación de Modelos Educativos (CIME) de México, basados en la solución de problemas y material didáctico, regletas de Cuisenaire y geoplanos que ayudan al estudiante a relacionar la Matemática con la realidad. Se busca además, equilibrar el qué se debe enseñar, el cómo el estudiante aprende y el para qué enseñar, tomando las concepciones y experiencias de la comunidad de aprendizaje, con el fin de alcanzar un término medio de los acercamientos de la enseñanza basado en prácticas psicopedagógicas actuales.

Parte de dicha investigación, que reporta este escrito, propone que los profesores desarrollen un conocimiento acerca del proceso afectivo, motivacional y de aprendizaje centrado en las característica de los estudiantes y cómo sus creencias, afectos, emociones y sentimientos, hacia la Matemática, la Clase de Matemática, aprendizaje matemático y estrategias de aprendizaje, influencian el proceso educativo de los alumnos. 


\section{Marco Teórico}

El proceso de enseñanza y aprendizaje de la Matemática se fundamenta en el rol activo del estudiante que construye nuevo conocimiento a partir de sus experiencias previas e implica una actividad meta-cognitiva constante, a través de la cual el estudiante se debe preguntar sobre la utilidad de lo que aprende, darse cuenta cómo y para qué aprende.

El desarrollo educativo de la investigación general, en la que no se profundizará en este artículo, está estructurado como parte de los conocimientos planificados para la asignatura y se definen como procesos de toma de decisiones intencionales en los cuales el alumno elige y recupera, de manera coordinada, los conocimientos que necesita para complementar una determinada demanda u objetivo y depende de las características de la situación educativa cuando se produce la acción (Monereo, Castello, Clariana, Palma, Pérez, 1995, Monereo, Pozo, Castelló, 2001). Dichos procesos son acciones organizadas que implican el conocimiento de técnicas, principios o reglas para entender un material o dominar una habilidad, integrar un nuevo conocimiento con el conocido y recordarlo en momentos adecuados. Los aprendices exitosos las emplean en forma flexible y contingente a la tarea de adquirir conocimiento, adaptan su uso y mantienen un control sobre su aplicación (Flores, 2001).Que los alumnos apliquen estrategias para su aprendizaje no es algo que surge espontáneamente, necesitan ser promovidas y enseñadas, el estudiante debe ser capaz de hacer dos tareas meta cognitivas: planificar y evaluar. En investigaciones anteriores mencionadas, se inició la formación de profesores, en la presente, se involucran a los alumnos para que, desde un marco integral, procuren el paradigma actual "enseñar a aprender, aprender a aprender o enseñar a pensar.

Para la enseñanza estratégica y aquella basada en la estrategia de solución de problemas la comunidad de aprendizaje estudió autores como Schoenfeld, 1999; Flores,1999, 2003, 2005(a), 2005 (b); Blum, 2002, Pisa, 2012, Mejia, Loango, 2014, cuyas tesis se enmarcan en un contexto donde los datos, relacionados dentro del texto de un problema, guardan una coherencia. Se toma como referencia también a Polya (1990), Schoenfeld (1992, 1999), quienes plantean que los problemas y algoritmos no deben ser entendidos en una relación secuencial unidireccional, sino como parte de un ciclo recursivo durante el proceso de solución.

La relevancia de las emociones en el proceso de aprendizaje de los estudiantes se analiza desde el enfoque de Forgas (2001); la perspectiva cognitiva social aporta argumentos que apoyan la comprensión de la construcción del conocimiento y las interacciones sociales del estudiante en el aula que influyen en el aprendizaje; demuestran que el afecto está íntimamente relacionado con todo lo que el estudiante piensa y hace. 
A partir del trabajo de MacCombs y Wistler (1997,2001), vigente en el paradigma psicopedagógico actual, se considera fundamental el enfoque del proceso de aprendizaje centrado en el estudiante. Es una perspectiva que proporciona información y dirige la toma de decisiones en el proceso educativo.

Los estudiantes continuamente procesan información de su entorno y de acuerdo con sus percepciones y experiencias van formando sus conclusiones con las que conforman sus creencias, las cuales las comparan con las de otras personas, las evalúan continuamente y las mantienen o modifican en el avance del proceso de aprendizaje. Las representaciones, opiniones, valores, emociones y actitudes de los estudiantes forman parte de sus creencias. (Hart 1989, Pajares, 1992, Latorre y Blanco, 2007,Damasio, 2011).

Schoenfeld (1992) y Clark (2008), siguiendo el trabajo del primero, estudiaron la interacción entre el dominio cognitivo y el afectivo, y la forma en que las concepciones de los alumnos sobre la Matemática delimitan cómo ellos se involucran en la actividad. Sus resultados indicaron que las ideas y creencias de los estudiantes acerca de la Matemática influyen en su participación, hábitos de trabajo, motivación y como resuelven sus problemas en el aprendizaje de la disciplina,

Se toman en cuenta los aportes de Goldín (2002), Gómez - Chacón (2003) y Mellado, et al(2014), quienes argumentan que la estructura de creencias que sustenta el pensamiento del estudiante hacia la Matemática, está relacionado con el afecto y meta- afecto, los que definen como un sistema de representación del individuo cognitivo y meta-cognitivo, ubicando este sistema en un dominio afectivo que lo dividen en emociones, actitudes y creencias. Puntualmente Gómez - Chacón argumenta que “ las creencias Garritz A., Mellado L., proporcionan significados personales y ayudan al individuo a atribuirle cierta relevancia a los distintos temas, como miembro de un grupo social“; si el estudiante no le da importancia y no entiende la Matemática, estudiarla le estresa y le produce emociones, sentimientos encontrados y su sistema de representación hará que no se sienta motivado a aprenderla.

Las creencias que tienen más influencia en los estudiantes son las concernientes a sí mismo, su relación con la Matemática y la disciplina tienen un fuerte componente afectivo e influyen en las creencias relativas a la confianza, auto-concepto y a la atribución causal del éxito o fracaso escolar. Estas creencias están estrechamente relacionadas con la meta-cognición y autoconciencia. (Lebrija, 2010) (2012). Con base en lo hasta ahora expuesto, se concluye que las creencias no se pueden cambiar fácilmente. Los educadores matemáticos que quieran modificar la estructura de las creencias de sus alumnos, no podrán tener éxito si solamente se centran en un discurso 
de cambio, o en un discurso donde se promueve que la matemática es útil y necesaria. Es importante proveer a los educandos de experiencias que sean suficientemente ricas, variadas, e importantes afectivamente, para promover que los estudiantes construyan nuevos afectos y meta - afectos.

Es por todo lo anterior, que dentro del marco general de la investigación, después de un año, los alumnos expresan sus experiencias con el programa. El presente artículo, se limita a exponer parte de la investigación, pues solo se reportan sus opiniones, de acuerdo con sus creencias hacia la Ciencia matemática, hacia la clase de Matemática, hacia el proceso de aprendizaje matemático y hacia las estrategias de aprendizaje utilizadas en el proceso educativo.

\section{Marco metodológico}

El presente artículo analiza los resultados parciales de la investigación general que dan respuesta al objetivo específico: Analizar las creencias acerca de las emociones-sentimientos-afectos de los estudiantes, hacia la Ciencia Matemática, Clase de Matemática, Aprendizaje matemático, estrategias de aprendizaje y su relación con el proceso de aprendizaje.

\section{Diseño de Investigación y tipo de estudio.}

El estudio de la investigación general utiliza un diseño de investigación mixto, cuali-cuantitativo, para medir el aprendizaje, las estrategias de aprendizaje, creencias de los alumnos hacia la Matemática; es un diseño de campo que permite medir los cambios que se dan durante el proceso educativo, un enfoque más práctico que teórico y auto-reflexivo durante el proceso. (Alvarez-Gayou, 2007, Hernández, 2004). Es una investigación acción, aplicada en un colegio privado de la Ciudad de Panamá, es exploratorio en el País pues no hay investigaciones sobre el tema a nivel pre-medio; es descriptivo, por lo que se detallan los logros acerca de las creencias y las estrategias utilizadas por los estudiantes (Baptista, Hernández, Fernández, 2008, Sabino, 2000).

De este proceso sistémico, el presente artículo está dedicado al análisis y desarrollo de los alumnos relativos a las estrategias de aprendizaje $\mathrm{y}$ a sus afectos, emociones y sentimientos durante un año escolar.

\section{Población, Sujetos y Tipo de muestra.}

La población del proyecto general tiene dos grupos estudio: docentes $\mathrm{y}$ estudiantes.

Los sujetos participantes son 93 alumnos-de séptimo nivel de premedia, quienes participan en el programa desde hace un año. No se describe el otro grupo estudio porque no será analizado en el presente artículo. Los alumnos fueron seleccionados al azar en donde todos tuvieron la misma 
posibilidad de participar en el estudio. El porcentaje de error de la muestra es de $5 \%$, con un nivel confianza del $90 \%$.

\section{Variables}

Variable I: Emociones - Sentimientos - Afectos hacia la Matemática

Definición conceptual: debido a la congruencia de la investigación con las propuestas teóricas consultadas se eligió elaborar una definición conjunta para las emociones, los sentimientos y afectos con los paradigmas de varios autores.

Las emociones son un conjunto complejo de respuestas químicas y neuronales que forman un patrón distintivo. Estas respuestas son producidas por el cerebro cuando detecta un estímulo emocionalmente competente, es decir, cuando el objeto o acontecimiento, real o rememorado mentalmente, desencadena una emoción y las repuestas automáticas correspondientes. Los sentimientos y afectos (meta-afecto), en cambio, son la evaluación consciente que se hace de la percepción de nuestro estado corporal durante una respuesta emocional. Los sentimientos son conscientes, objetos mentales como aquellos que desencadenan la emoción (imágenes, sonidos, percepciones físicas). Las emociones que no se perciben como sentimientos son inconscientes, sin embargo, pueden tener efecto sobre nuestras conductas. (Monge, 2009)

Definición operacional: Se miden las emociones - sentimientosafecto hacia: la Matemática, hacia el aprendizaje de la Matemática- y hacia la clase de Matemática.

Variable II: Creencias hacia la Matemática, su enseñanza y su aprendizaje.

Definición conceptual: Creencias son verdades personales indiscutibles sustentadas por cada uno, derivadas de la experiencia o de su entorno que tienen un fuerte componente evaluativo y afectivo. Las creencias se manifiestan a través de declaraciones o acciones. (Pajares, 1992).

Definición operacional: Las creencias se miden de acuerdo con cuatro criterios: creencias hacia la Matemática, hacia la Clase de Matemática, hacia el proceso de aprendizaje de la Matemática, hacia las estrategias de aprendizaje.

Variable III: Estrategias de aprendizaje.

Definición conceptual: Las estrategias de aprendizaje, son el conjunto de actividades, técnicas y medios que se planifican de acuerdo con las necesidades del estudiante, con respecto a la tarea educativa a realizar, todo esto con la finalidad de hacer más eficiente el proceso de aprendizaje. (Sáenz, Lebrija, 2014). 
Definición operacional: Se medirán las cuatro estrategias de aprendizaje más relevantes para el proceso Matemático: comprensión lectora, toma de apuntes, resolución de problemas, estrategias de estudio.

\section{Instrumentos}

- $\quad$ Cuestionario tipo Likert para las creencias y estrategias de aprendizaje de los alumnos.

- $\quad$ Cuestionario abierto para medir las creencias de los alumnos sobre la clase de Matemática, hacia el método de enseñanza, el aprendizaje y las emociones-sentimientos hacia la Matemática, elaborado y validado por la comunidad de aprendizaje del Proyecto.

- $\quad$ Cuestionario abierto para medir el conocimiento de los estudiantes sobre sus estrategias de aprendizaje.

- Grupos focales con alumnos sobre el proceso de enseñanza y aprendizaje y sus emociones y sentimientos hacia el proceso.

- $\quad$ Cuestionarios de opinión alumnos.

2.5 Procedimiento. Se realizaron talleres de trabajo con la comunidad de aprendizaje para analizar la problemática de la enseñanza y aprendizaje de los alumnos en la asignatura de Matemática.

- La comunidad educativa propuso analizar las creencias de los estudiantes mediante 4 temáticas relevantes: la importancia de las creencias de los estudiantes hacia la clase y la Ciencia Matemática, el método educativo, las estrategias y el proceso de aprendizaje matemático; utilizó para ello un cuestionario de preguntas abiertas que permitió que los alumnos se expresaran libremente, además del desarrollo de grupos focales (Aubel, 1994).

- $\quad$ Los grupos focales se desarrollaron simultáneamente en los 3 salones de séptimo de premedia, ingresando dos investigadores por grupo, se fueron realizando con las 4 temáticas propuestas Los estudiantes fueron aportando sus ideas y se fueron escribiendo en el tablero. Una vez culminado el ejercicio en cada salón, se procedió al análisis de los datos para organizar en categorías los datos obtenidos.

- $\quad$ Además de los resultados de los grupos focales, para evaluar el afecto de las emociones y los sentimientos de los educandos hacia el aprendizaje, la clase y la Ciencia Matemática se utilizaron los cuestionarios de preguntas abiertas y escala tipo Likert, con respuestas de totalmente en desacuerdo a totalmente de acuerdo. Estos últimos cuestionarios fueron validados a través de jueces expertos, analizando claridad del reactivo, pertinencia, redacción y relevancia.

- Para establecer las categorías de los instrumentos de preguntas abiertas, primero se revisaron dichas preguntas, posterior a eso se procedió a 
buscar patrones generales de las respuestas similares o comunes de los cuestionarios, por último, se clasificaron las respuestas para analizarlas.

- $\quad$ Las estrategias de aprendizaje de los estudiantes se valoraron a través de cuestionarios abiertos; se les pidió que describieran las estrategias de aprendizaje que utilizaban en el salón de clase; posterior a ello, cómo realizaban las estrategias de comprensión lectora, toma de apuntes, solución de problemas y de estudio, que fueron mencionadas en la pregunta inicial.

- Obtenidos los datos, se hizo el análisis estadístico descriptivo, obteniendo los porcentajes de respuesta de los estudiantes antes y después del curso escolar, lo que permitió evaluar las creencias de los estudiantes durante el proceso de implementación del modelo educativo de enseñanza y aprendizaje de la Matemática.

\section{Resultados.}

En esta primera etapa de la investigación se tienen resultados de la intervención de la comunidad de aprendizaje, analizados con estadística descriptiva, los porcentajes de las creencias de los participantes tomando en cuenta los cuatro criterios ya descritos

\section{Ciencia Matemática}

Se pueden lograr cambios innovadores en las aulas de clase, pero si no se trabaja con la forma de pensar de los estudiantes el avance es menor o nulo.

Una de las razones por las cuales el grupo investigador considera que a los estudiantes no les gusta la Matemática es que no le ven la utilidad en su vida cotidiana. Así, los estudiantes perciben la Matemática solo en el contexto académico y por ello lo que les preocupa es sacar malas notas ligado a no comprender los temas expuestos en clase. Por eso se les preguntó "les gusta estudiar Matemática”. Las respuestas las podemos observar en la gráfica 1.

\section{Me Gusta estudiar Matemática}

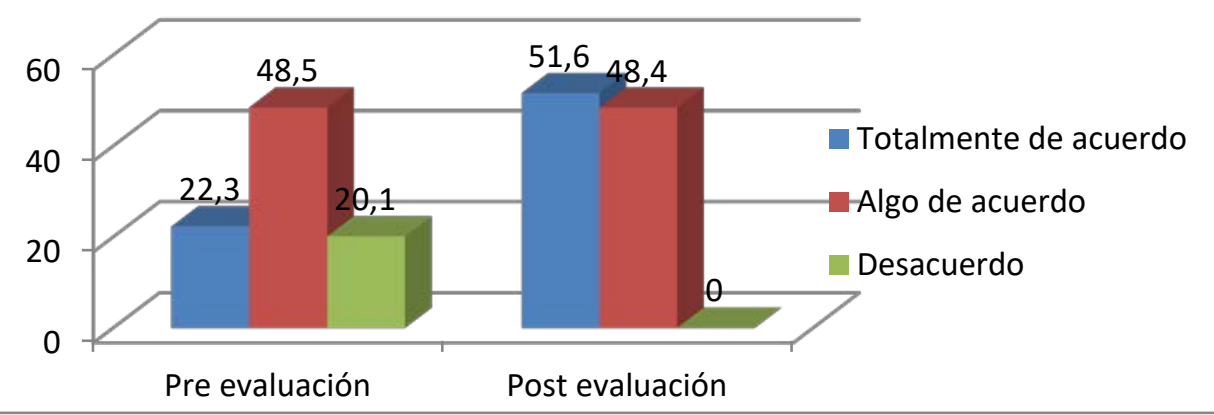

Gráfica 1 
Se considera que si el alumno estudia Matemática encontrándole la relación del contenido académico con sus problemas diarios y su entorno, según la literatura consultada, varían sus valores, emociones y actitudes y podría estudiar la disciplina con mayor gusto y tener mejor disposición al enfrentarse al conocimiento.

En la evaluación se observa un cambio en el primer año de intervención dentro del salón de clase. Esto se logró con la retroalimentación directa entre investigadores y profesores dentro y fuera del salón de clase. Fuera del salón de clases en talleres con los docentes, dentro del salón el grupo investigador ha logrado una sinergia positiva entre todos los interventores: investigadores, profesores alumnos y administrativos del colegio.

\section{Clase de matemática}

Analizar lo que ocurre dentro del aula es fundamental para tener un proceso educativo de calidad y conocer las creencias de los estudiantes que pueden potenciar u obstaculizar el proceso. Por ejemplo, es costumbre entre los estudiantes no exponer dudas ante sus compañeros, que se puede mejorar en un ambiente de confianza y diálogo con ellos. Por ello, la comunidad de aprendizaje consideró preguntar "le gusta la clase de Matemática”. La gráfica 2 muestra el cambio que se produjo en el año.

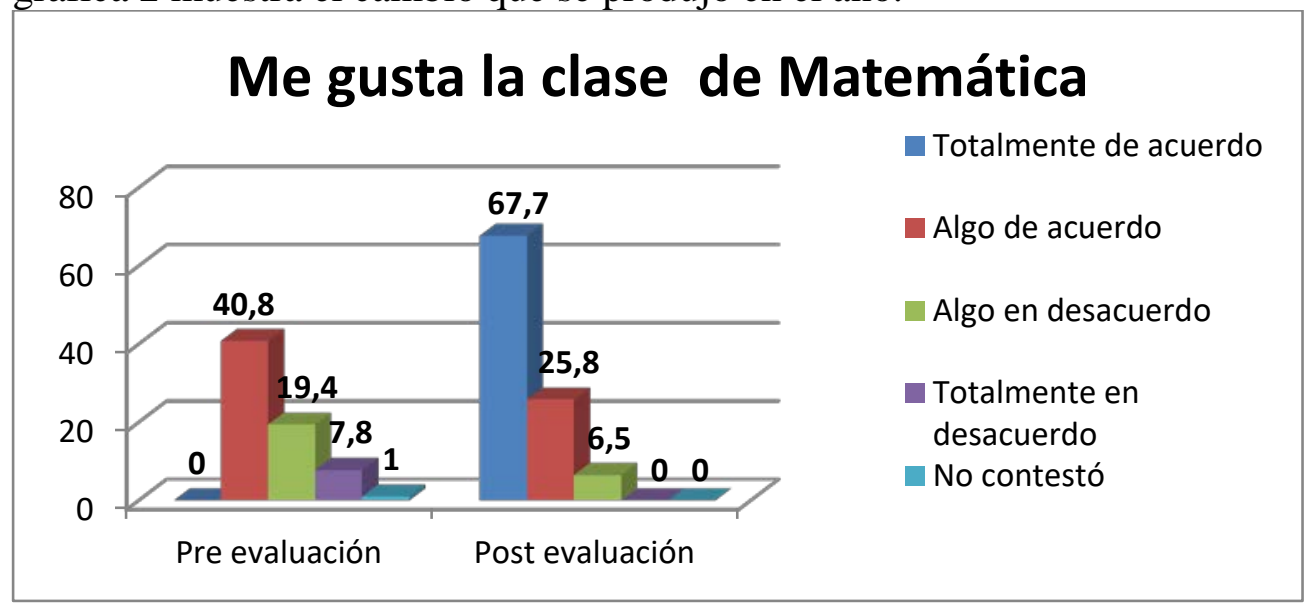

Gráfica 2

Inicialmente a nadie le gustaba totalmente la clase de Matemática. Se muestra un cambio después de un año de intervención, lo que indica que el proceso de enseñanza y aprendizaje propuesto es adecuado, positivo y motiva a los estudiantes a aprender.

Igualmente, es importante comprender cómo se sienten los estudiantes en su clase de Matemática para valorar la aplicación del modelo educativo que fomenta las estrategias de aprendizaje, utiliza material lúdico 
y promueve la motivación, entre otros aspectos didácticos relevantes. Para ello se les indicó lo siguiente.

Subraye la palabra que indique como se siente en la clase de Matemática: (los estudiantes pueden expresar más de una respuesta). Los resultados están en la gráfica 3.

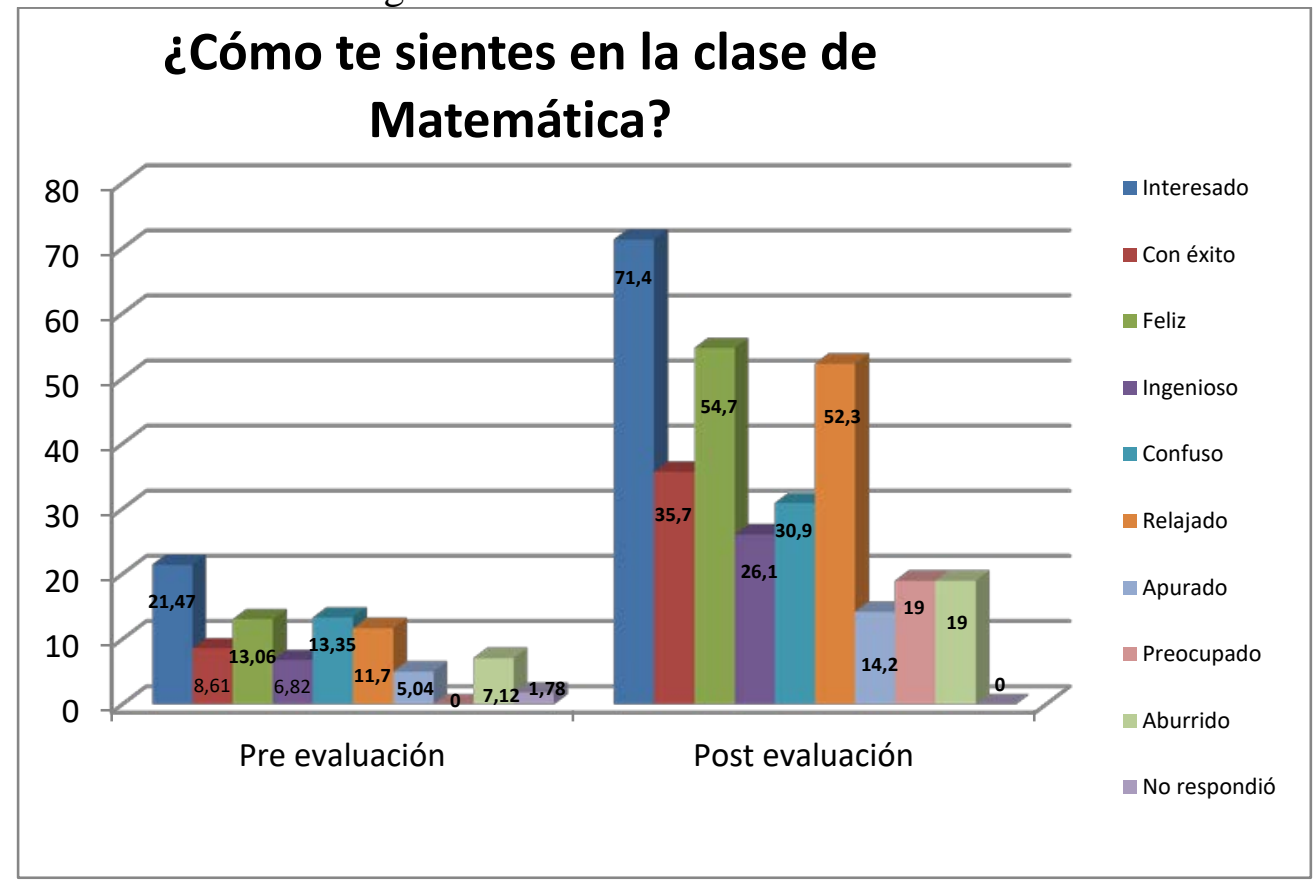

Gráfica 3

Los sentimientos experimentados son positivos y negativos; en la gráfica muestra que se expresan interesados, con éxito, feliz, ingenioso, relajado pero también hay quienes se sienten aburridos; hay expresiones de confuso, apurado, preocupado que deben seguirse analizando para resolverlos, porque este tipo de sentimientos son causas de emociones y conductas negativas dentro del salón de clases como desatención, inquietud e indisciplina que alteran el ambiente de la clase. Se ve igualmente que al inicio hay quienes no contestaron, pero en la segunda evaluación expresaron algún sentimiento.

Dentro del mismo cuestionario se les indicó "anote algún otro sentimiento en clase de Matemática”, (los alumnos podían dar más de una respuesta). Los resultados se pueden observar en la gráfica 3’. 


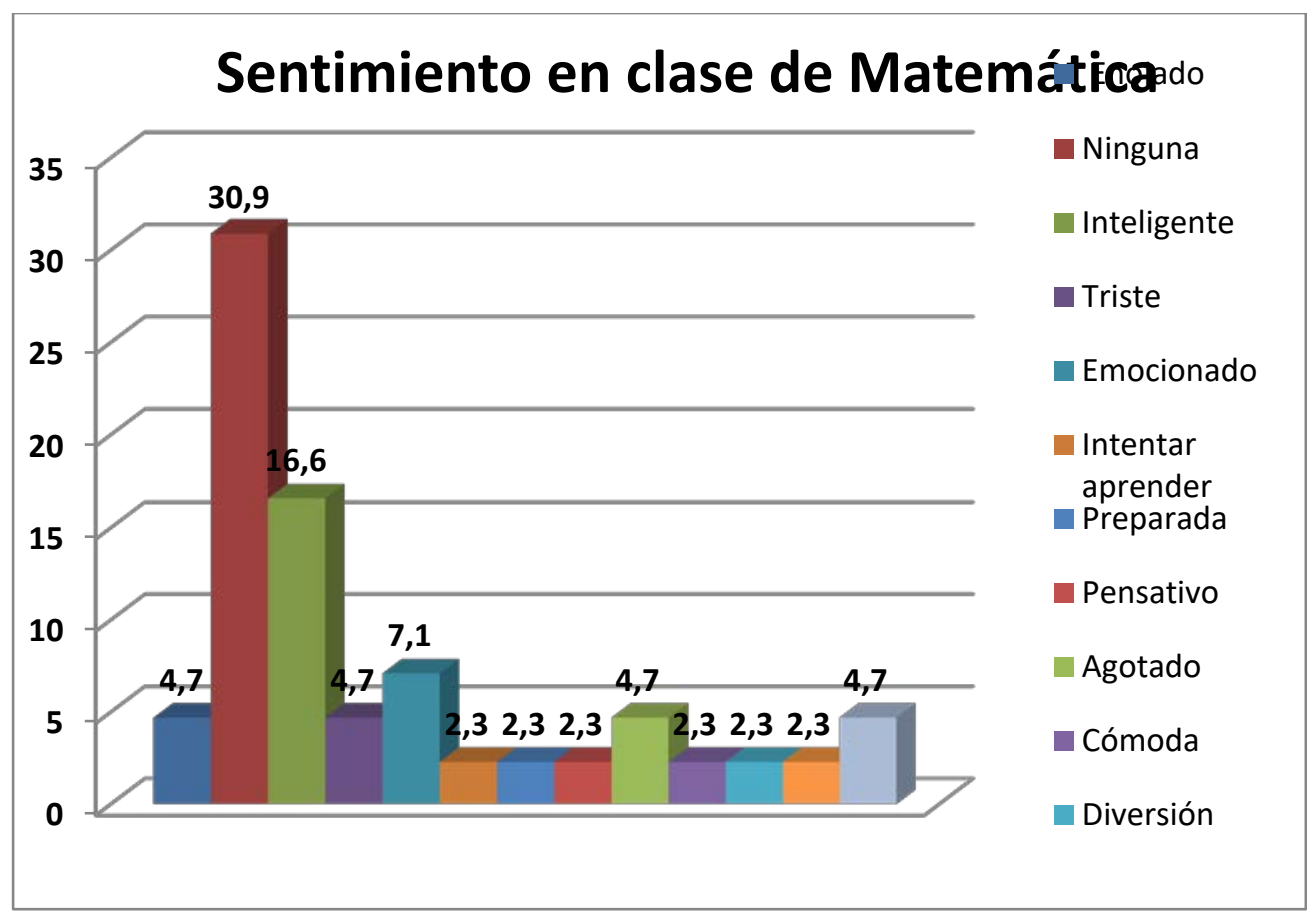

Gráfica 3’

Resalta el hecho de que una mayoría no expresó otro sentimiento adicional al de los expresados en la gráfica 3, aunque si hubo otros sentimientos como el de calificarse como inteligentes y emocionados en la clase. Concluimos con esto que la mayoría se siente capaz y gustosos de aprender Matemática. Son pocos los estudiantes con otros sentimientos desfavorables, todos son interesantes y se van a seguir analizando más a fondo y más general.

\section{Aprendizaje de la Matemática.}

Para la comunidad educativa es importante erradicar la creencia entre los alumnos de que "no todos tienen la inteligencia para aprender Matemática”. El cambio en sus opiniones influiría en su interés y motivación hacia su aprendizaje. La gráfica 4 muestra la creencia de los estudiantes al respecto. La pregunta fue ¿ंTodos los estudiantes pueden aprender Matemática? 


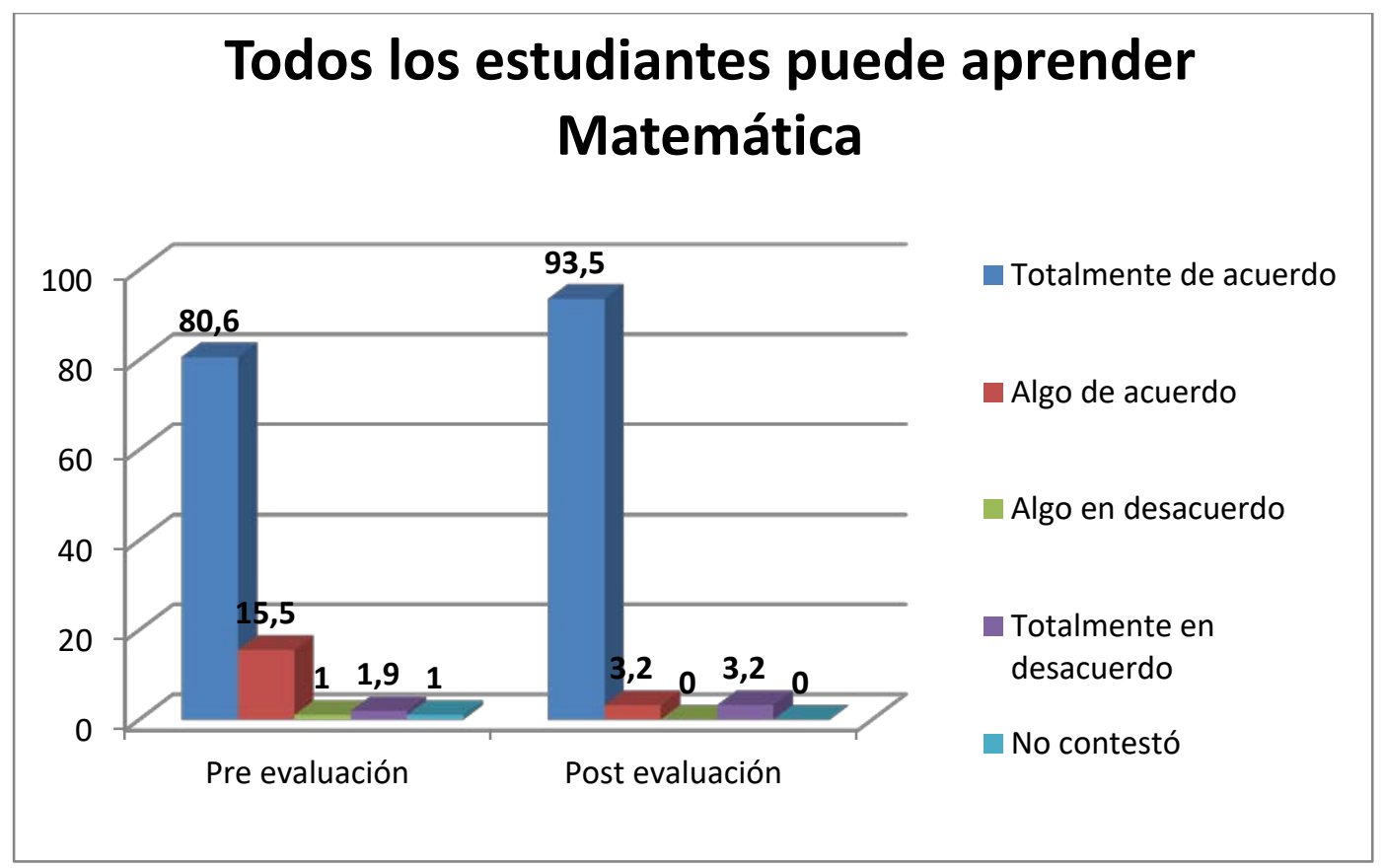

Gráfica 4

Desde el inicio, un buen porcentaje de estudiantes manifestaron que pueden aprender Matemática, evaluación positiva al respecto; pero en la segunda evaluación este porcentaje se incrementó positivamente; en la segunda medición bajó el "algo en desacuerdo", pero creció el porcentaje de "totalmente en desacuerdo", y aunque es bajo el cambio el porcentaje es una información que debe ser estudiada; lo anterior se puede aunar al hecho de que en la segunda medición todos contestaron; esto último muestra confianza en el ambiente de aprendizaje. En la medida que se sepa que todos los alumnos tienen la creencia que pueden aprender Matemática se facilitará la enseñanza.

\section{Estrategias de aprendizaje.}

Las estrategias de aprendizaje permiten la organización, hábitos de estudio y fomentan la autonomía necesaria para un proceso de aprendizaje con significado, por ello, la comunidad de aprendizaje considera que son tan importantes como el conocimiento de los contenidos matemáticos; piensa que el dominio de tales estrategias permite la reconstrucción del conocimiento sin la intervención del docente. Se preguntó a los estudiantes” qué estrategias de aprendizaje utilizas en tus clases de Matemática” (los alumnos podían contestar más de una respuesta). En la gráfica 5 se muestran las estadísticas. 


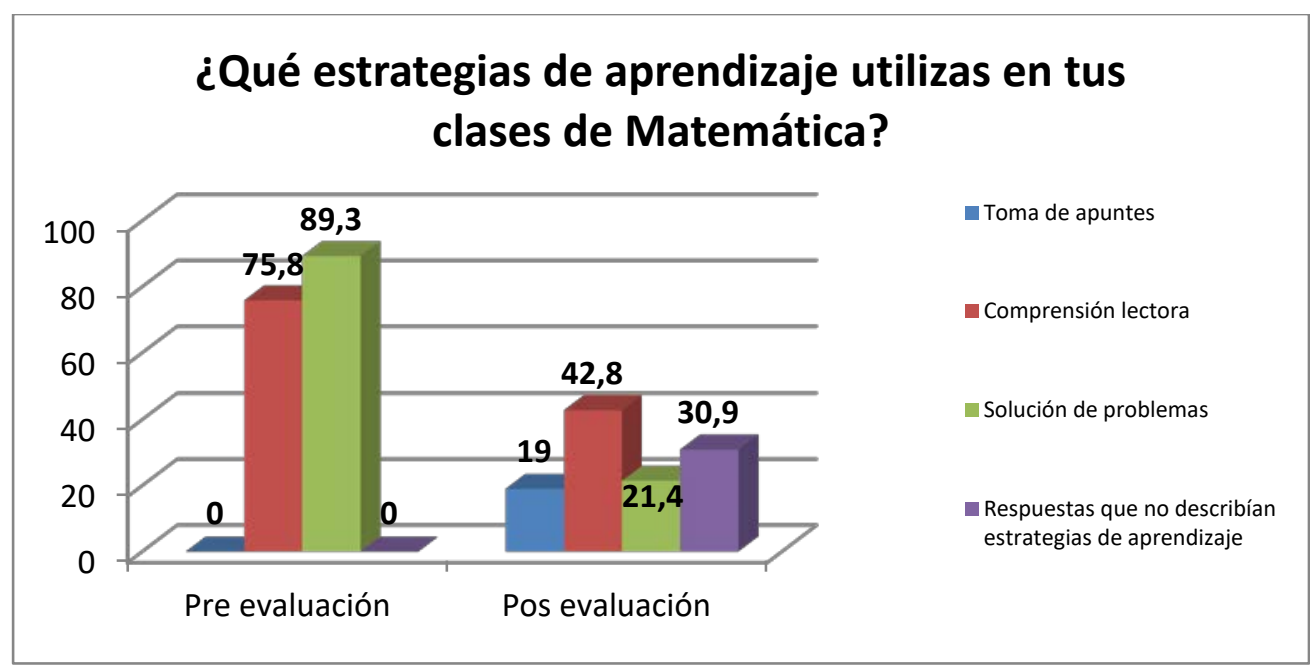

Gráfica 5:

La pre evaluación indica que utilizan la estrategia de comprensión lectora, la cual es fundamental para la comprensión de los problemas reales, actividad didáctica estructural del modelo educativo de la investigación general, que desarrolla un proceso de aprendizaje autónomo y adecuado. Los estudiantes mencionan la utilización de la estrategia de solución de problemas que como construcción cognitiva y metacognitiva, se considera muy positiva dentro del proceso de enseñanza aprendizaje que se promueve. Sin embargo, en el salón de clases, después de esta medición, cuando se preguntó a los alumnos cómo realizan las estrategias, el $69.8 \%$ de los estudiantes desconocían realmente la estrategia de comprensión lectora, el $50.9 \%$ escribe los apuntes del tablero, sin pensar en tomar las ideas principales de lo que se explica en clase, que no es solamente copiar; solo el $17 \%$ de los estudiantes lograron explicar adecuadamente cómo realizan la estrategia de solución de problemas, lo que implica que la aplican y saben cómo usarla, el resto se ocupó solamente en encontrar la solución del problema propuesto; posterior a ello, se retroalimentó al profesor para que la enseñara y lograra que sus alumnos la interiorizaran. La evaluación realizada posteriormente arrojó mediciones que muestra la gráfica 5. Se está trabajando en las creencias de los estudiantes de que el conocimiento académico es tan importante como el aprendizaje de las estrategias para "aprender a aprender". Antes de particularizar las estrategias, en una encuesta separada, el $55,2 \%$ de los estudiantes manifestaron que las estrategias no tenían importancia para un aprendizaje duradero

La información anterior se amplió utilizando la técnica de grupos focales (focus group) que nos permitió profundizar la interpretación de los datos obtenidos con el cuestionario de creencias. Hubo mayor cantidad de 
respuestas que enriquecieron la información para redireccionar el desarrollo de la propuesta dentro del salón de clases. La técnica se desarrolló en un ambiente de seguridad, sin autoridades, de manera que los estudiantes pudieron expresarse de forma espontánea y con la participación de todos los participantes. En el avance del proceso, la comunicación colaborativa nos permitió mejorar dicha información

El calendario académico en Panamá comienza en el mes de marzo y concluye en el mes de diciembre, culminando, haremos una nueva evaluación para conocer los logros para la investigación general.

\section{Conclusion}

Al analizar las creencias de los estudiantes sobre sus emocionessentimientos-afectos y su relación con el proceso de aprendizaje, se están observando posibilidades de cambio en el aprendizaje de los estudiantes basado en las reflexiones, análisis, retroalimentación y seguimiento en la práctica docente y estudiantil cotidiana en búsqueda de un aprendizaje autónomo. La retroalimentación se desarrollaría entre profesores, alumnos e investigadores con talleres y seminarios. A manera de ejemplo exponemos la guía de observación de clase que ayudaría para la investigación general. (Ver ANEXO).

A partir de estos resultados incipientes se tienen más elementos para resaltar la importancia del acompañamiento durante el curso escolar.

Observamos la necesidad de que los cursos de formación para estudiantes se estructuren de las necesidades y opiniones del alumno y no solo de las sugerencias de expertos, muchas veces ajenos a la problemática real en el aula.

Somos de la opinión que la participación de comunidades de aprendizaje en continua comunicación con los alumnos in situ permite el diálogo pertinente para alcanzar logros conjuntos.

La discusión y análisis entre pares y expertos en los talleres y la utilización de la técnica de grupos focales que se han ido desarrollando permiten exponer fortalezas y debilidades que al trasladarse al salón de clases para ponerlas en práctica traerá avances en el aprendizaje de los alumnos.

Los argumentos que amparan el desarrollo de la propuesta es esta primera medición, fundamental para lograr el objetivo general de la investigación; esta evaluación muestra que se están alcanzando cambios en las emociones, sentimientos y afectos de los estudiantes que opinamos afectan positivamente sus actitudes para escuchar, estudiar y sobre todo preguntar las dudas que generalmente no se atreven a hacerlo, participan sin tanto estrés de la clase y propiciará un ambiente de diálogo y motivación para lograr el aprendizaje necesario. 
Con base en los datos obtenidos concluimos que los estudiantes modificaron sus creencias positivamente hacia la Matemática; que los educandos mencionen que les gusta, es el primer paso para lograr el interés y la motivación intrínseca hacia el estudio de esta Ciencia y que por la falta de comprensión de cómo utilizarla, en los problemas cotidianos, existen emociones negativas que obstaculizan el involucramiento de los estudiantes en su comprensión y estudio. (Gráfica 1, pp. 7).

Otro de los aspectos que se pudo comprobar es la importancia de las relaciones, cercanas y empáticas entre profesor y alumno. Si bien es cierto que se debe estructurar la clase en reglas y disciplina adecuada para la concentración y construcción del conocimiento, esto no implica que el docente sea autoritario y no sepa establecer una comunicación asertiva y recíproca con sus estudiantes. (Gráficas 2 y 3 pp. 8).

Los resultados reportan que los estudiantes tienen un interés similar hacia el aprendizaje de la Matemática y la clase de Matemática. La modificación en sus creencias va a favorecer su aprendizaje. (Gráficas 1 y 2 pp. 7 y 8 ).

Una de las creencias más difíciles de modificar es "que la Matemática es para todos y no sólo para unos cuantos”, la evaluación nos mostró un cambio positivo en esta creencia. (Gráfica 4, pp.10).

Se deben seguir profundizando en el conocimiento de las estrategias de aprendizaje para posteriormente medirlo y trabajar en su uso. La estrategia de comprensión lectora, para desarrollar las competencias de redacción, de organización de la información; tomar buenos apuntes y sintetizar la información de los libros. Esta estrategia potencia la de solución de problemas cotidiano, objetivo general de la investigación, que aumenta el interés por el conocimiento de la Ciencia. Las últimas investigaciones indican que es insuficiente enseñar técnicas a los alumnos que no vayan acompañadas de un uso estratégico, esto es, la utilización de metaconocimiento. La repetición ciega y mecánica de ciertas técnicas no supone una estrategia para el aprendizaje. (Gráfica 5 pp. 10).

La comunidad de aprendizaje ha discutido y se ha involucrado con los participantes de la investigación acerca de estos criterios; concluye que el proceso de aprendizaje autónomo y auto-regulado del estudiante se promueve a través de la enseñanza de estrategias de aprendizaje, pero la utilización de éstas, están ligadas con la motivación y sentir de los estudiantes no solo hacia la Ciencia Matemática, sino hacia el proceso de aprendizaje en general. Cuando el estudiante modifica las creencias negativas hacia la Matemática y su aprendizaje, se motiva, valora su conocimiento y se interesa en aprenderla de otra manera con mayor beneficio. 
En definitiva, se considera que para poder producir un cambio en la percepción de los estudiantes hacia la Matemática hay que modificar las creencias, relativas a las emociones, sentimientos y afectos errados que tienen sobre esta Ciencia, el aprendizaje debe estar ligado directamente con la utilidad para el estudiante y por tanto, su importancia en el entorno inmediato. Este cambio coadyuvará a reducir los altos índices de reprobación escolar en la disciplina, aminorar el miedo que se le tiene, pero sobre todo a promover su comprensión y utilización.

Por tanto, las creencias del educando están presentes en el proceso de aprendizaje y no se puede dejar de lado toda esta estructura que es la que permite que el estudiante tenga un rol activo en su proceso educativo.

Además de las mediciones anteriores se tienen avances de evaluaciones que actualmente se desarrollan en torno al diseño y ejecución del programa que propone la investigación general. Inicialmente se tienen cifras como las que siguen. Un 48,5\% de estudiantes manifestaron que no se pueden cometer errores porque de ellos no se aprende y se tienen malas calificaciones, situación que debe revertirse pues los fracasos dejan consecuencias que pueden ser aprovechadas; se ha conversado mucho con el profesor acerca de que un 43,7\% de los estudiantes dicen que rinden más en sus clases de Matemática cuando los profesores promueven la expresión de lo que piensan y sienten $\mathrm{y}$, siembren relaciones afectuosas; de esto se concluye que es importante desarrollar la empatía y el trabajo colaborativo con los estudiantes, para promover el aprendizaje significativo; sin embargo, un 62,1\% de los estudiantes están de acuerdo con que la clases funciona mejor si el profesor es autoritario; esto proyecta que los alumnos mantienen creencias fundamentadas en paradigmas educativos en donde el proceso está centrado en el profesor y a través de estrategias directivas desarrolla el aprendizaje. Es importante la comunicación y el trabajo consensuado para que los estudiantes sean activos, participen y construyan el conocimiento por convicción; el 35,9\% de los estudiantes opinan que es importante que el profesor tome en cuenta sus necesidades sociales, emocionales y físicas, más que las intelectuales.

La respuesta coincide con el paradigma psicopedagógico actual de que el aprendizaje debe estar centrado en el alumno y en las características de los educandos para promover el aprendizaje útil. Estamos por medir nuevamente y establecer el cambio. Se resume que los educandos deben aprender a aprender, conocer sus fortalezas y debilidades para poder organizarse mejor y lograr un aprendizaje asertivo.

\section{Limitaciones y dificultades en el cambio de las prácticas estudiantiles.}

El análisis, previo a la conclusión de la investigación, indica que la formación sea realizada dentro de un proceso largo de intervención. Aunque 
los adolescentes pueden cambiar sus creencias con mayor facilidad que los adultos, si tienen la figura que admiran y sea modelo de sus objetivos, no es suficiente hacer recomendaciones, hay que “enseñar aprender a aprender”.

El estudiante debe sentir la enseñanza dirigida a su aprendizaje y esto no se logra con rigidez e incomprensión hacia sus necesidades. Esto tampoco es sencillo proyectarlo en las clases. Cada estudiante tiene sus diferencias individuales de aprendizaje y hay que considerar que esto es importante para que el alumno se sienta co-creador de su aprendizaje.

Durante mucho tiempo los profesores se han preocupado fundamentalmente por la transmisión de los contenidos de sus asignaturas. Muchos profesores enseñan las estrategias de aprendizaje pero sin relacionarlas con los contenidos de las materias. Para estos profesores, los alumnos serían capaces por sí mismos de aplicarlas sin necesidad de una intervención educativa que promueva su desarrollo o aplicación. Por ello en la comunidad se trabajan estos inconvenientes que pueden dar al traste con la iniciativa.

Como parte de la educación Matemática el modelo ha promovido la enseñanza de estrategias de aprendizaje, que permiten al estudiante comprender cómo estudiar, cómo organizarse y ser autónomo para lograr su aprendizaje, esta necesidad no es sencilla de proyectar.

\section{References:}

1. Álvarez-Gayou,J.(2007). Cómo hacer investigación cualitativa. Fundamentos y metodología. Barcelona: Paidós.

2. AubeL, J. (1994)Guidelines for studies using the group interview technique. Ginebra; OIT

3. Hernández,R.(2008). Metodología de la investigación. México: McGraw-Hill.

4.Blum,W. (2002). ICMI study14: Applications and modeling in Mathematics education, discussion document Educational studies in mathematics 51,149-171.

5.CIME La información de esta referencia la encontrará en: http://www.cime.edu.mx/

6.Clark,C.,(1908). Asking the Right Questions About Teacher Preparation: Contributions of Research on Teacher Thinking. Educational Researcher, 17 (2), 5-12.

7.UNESCO (2005) Estudio regional comparativo y explicativo (TERCE): $\quad$ Recuperado el 15 de febrero 2016:www.unesco.org/new/es/.../third-regional-comparative-andexplanatory-study-terce/

8.Damasio, A.,(2011). El error de Descartes: la emoción, la razón y el cerebro humano. ISBN 9788423344963. Editorial Destino, 
Actualizado 2016.

9.Forgas, J. (2001). The affect infusion model: an interrogative theory of mood effects on cognition and figment, en Martin E., Clore G. Theories of mood and cognition: A users guidebook. Nueva Jersey: Lawrence Erlbaum Associates.

10. Flores, R., (1999). La enseñanza de una estrategia de solución de problemas a niños con problemas de aprendizaje. Integración, Educación y Desarrollo psicológico, 11,11. 1-17.

11. Flores, R., (2001). Instrucción estratégica en alumno con problemas de aprendizaje. Revista Mexicana de Psicología.

12. Flores, R., (2003). El conocimiento matemático en problemas de adición y sustracción: Un estudio sobre las relaciones entre conceptos esquemas y representación. Aguascalientes: Universidad Autónoma de Aguascalientes.

13. Flores, R.,(2005a). Aprendizaje de la Matemática: una perspectiva cognoscitiva. Conferencia para profesores y alumnos de Matemática de la Facultad de Ciencias de la Universidad de Panamá.

14. Flores, R.,(2003). El conocimiento matemático en problemas de adición y sustracción: un estudio sobre las relaciones entre conceptos, esquemas y representación. México. Ediciones de la Universidad Autónoma de Aguascalientes.

15. Flores, R,.(2005b). El significado del algoritmo de la sustracción en la solución de problemas. Educación Matemática, 17,7-34.

16. Gómez, I.(2003). La tarea intelectual en Matemática. afecto, meta-afecto y los sistemas de creencias. Boletín de la Asociación Matemática Venezolana, ISSN-e 1313-4125, 10 (2), pp.223-248.

17. Goldin, G.,(2002). Affect, meta-affect, and mathematical belief structures. En Leder, G., Pehkonen, E., Torner, G., (2002) Beliefs: a Hidden Variable in Mathematics Education. Londres: Kluwer Academic Publisher.

18. Hart, L., (1989). Describing the affective domain: saying what we mean. En McLeod, D., Adams, V..(1989) Affect and mathematical problem solving: Nueva York. Spinger - Verlarg.

19. Hernández, R., (2004) Metodología de la Investigación. México: McGraw-Hill.

20. Latorre, M., Blanco, F.(2007). Algunos conceptos clave en torno a las creencias de los docentes en formación. Docencia e Investigación, 17, 147-170.

21. Lebrija, A., Trejos M., (2005). Matemática Creativa: Estrategias de Enseñanza Aprendizaje en el Aula. VIII Congreso Internacional de Investigación en el Aula, Facultad 
de Ciencias de la Educación. Universidad de Panamá.

22. Lebrija, A., Flores, R., Trejos, M., (2006). Influences of the teachers' beliefs and strategies in the teaching - learning process of math: a constructivist solution proposal. International Congress of Mathematicians. 22-30. Madrid, España.

23. Lebrija; A.,(2006). Problemas de aprendizaje en la adolescencia: Experiencias en el Programa Alcanzando el éxito en Secundaria. Capítulo de Libro, Facultad de Psicología, UNAM - aval CONACYT.

24. Lebrija, A., Flores, R., Trejos, M. (2010). El papel del maestro, el papel del alumno: un estudio sobre las creencias e implicaciones en la docencia de los profesores de Matemática en Panamá. Educación Matemática, 22 (1), 31-55.

25. Lebrija, A. (2010). Programa de Formación Continua para el Profesorado de Matemática: Desde un Enfoque de Enseñanza Centrado en el Alumno (tesis doctoral). Universidad Autónoma de Madrid, España.

26. Lebrija, A. (2012). Psicopedagogía aplicada a la enseñanza matemática. Revista Universidades. ISSN 0041-8935, No 53, págs. 40-50.

27. Mellado,V., Borrachero, A., Brígido, M., Melo, L., Dávila, M., Cañada, F., Conde, M., Costillo, E., Cubero, J., Esteban, R., Martínez, G., Ruiz, C., Sánchez, J., Garritz, A., Mellado, L., Vázquez, B., Jiménez, R. y Bermejo, M. (2014) Las emociones en la enseñanza de las ciencias. La enseñanza de las ciencias, 32, 11-36

28. Mejia, A., Loango M. (2014) Resolución de problemas matemáticos para fortalecer el pensamiento numérico en estudiantes del grado septimo de la institucion educativa adventista del municipio de Puerto Tejada Cauca. Cuba: Universidad de Católica de Manizales.

29. McCombs, B., Whistler, J. (2001) What do we know about learners and learning? The learner - centered framework: Bringing the educational system into balance. Educational Horizon, 79 (4) 182193.

30. McCombs, B., Whistler, J. (1997) Learner - Centered classroom and school. San Francisco: Jossey - Bass Publishers.

31. Monereo, C., Castello,M., Clariana, M.,Palma, M., Pérez, M. (1995). Estrategias de enseñanza $\mathbf{y}$ aprendizaje. Formación del profesorado y aplicación en la escuela. Barcelona, España: Graó.

32. Monereo, C., Pozo, J., y Castelló, M. (2001). La enseñanza de estrategias de aprendizaje 
33. en el contexto escolar. En Coll, C.; Palacios, J; Marchesi, A. (Comps.). Desarrollo

34. Psicológico y educación II (Edición revisada). Madrid: Alianza.

35. Monge, S.,(2009), Diferencia entre emoción y sentimiento. Consultado el 2 de febrero del 2015, de http://neuromarca.com/blog/diferencia-entre-emocion-y-sentimiento/

36. OECD (2010). PISA 2009 at a Glance. Recuperado el 2 de abril de 2016 de

37. http://www.oecd.org/pisa//46660259.pdf

38. PREAL (2007). Mucho por hacer. Informe de Progreso Educativo de Centroamérica y la República Dominicana. Recuperado el 15 de febrero de 2016 de http://www.oei.es/quipu/preal_info07.pdf

39. Pajares, F.,(1992). Mathematics Teachers Professional Knowledge, en J. Ponte, y J. Matos (Eds) Proceedings of the eighteenth international conferences for the psychology of mathematics education. Lisboa: International Group for the psychology of mathematics education.

40. Pisa (2012) Resolución de problemas de la vida real, Recuperado el 11 de enero, 2016:

41. http://www.mecd.gob.es/dctm/inee/internacional/pisa2012resolucionproblemas/pisa2012cba-1-4-2014web.pdf?documentId=0901e72b8190478c

42. Polya, G. (1990). Cómo plantear y resolver problemas. México: Trillas.

43. Underhill, R. (1988). Mathematics Learners beliefs:a review focus on learning problems in mathematics. Learning Problems in Mathematics.

44. Sabino, C. (2000). El Proceso de Investigación. Caracas. Panapo.

45. Sáenz C., Lebrija A. (2014). La formación continúa del profesorado de Matemáticas: una práctica reflexiva para una enseñanza centrada en el aprendizaje. Revista Latinoamericana de Investigación en Matemática Educativa editada por el Comité Latinoamericano de Matemática Educativa (RELIME), México. Vol. 17, No. 2, julio 2014 - $\quad$ pp. 219-244 - DOI: 10.12802/relime.13.1724.

46. Shoenfeld, A. (1992). Learning to think mathematically: Problem solving, metacognition, and sense making in mathematics. Documento disponible en internet en: http://www.rieoei.org/deloslectores/203Vilanova. PDF.

47. Schoenfeld, A. , Alan H. (1999)). Examining the Complexity of Teaching. Special issue of the Journal of Mathematical Behavior, 18 (3). 
Anexo

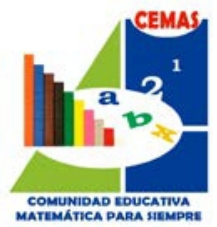

Matemática constructiva

Guia de observación en clase para el acompañamiento de la docencia

Escuela Grupo

Fecha

Educador(a) Tema

El objetivo de esta guía de observación es establecer una buena comunicación entre docentes matemáticos con el fin de mejorar la enseñanza y promover el pensamiento estratégico (estrategias de enseñanza y aprendizaje). Con la participación grupal se logrará el aprendizaje de los estudiantes que todos los que están insertos en el sistema educativo deseamos. El educador al planear su clase debe tener en cuenta estrategias metacognitivas como el análisis de su docencia y del por qué, cómo, cuándo y dónde es útil el contenido de cada tema. Tanto el docente como el estudiante deben establecer metas en cada clase. Proponemos para esto el enfoque constructivista que refleja la intención del educador de que sus alumnos descubran y construyan los conceptos $\mathrm{y}$, las relaciones matemáticas, a través de:

- $\quad$ el manejo de los materiales, de los ejercicios, juegos y problemas que plantea

- la creación de “conflictos cognitivos”, siembren dudas, cuestionen a sus alumnos para que defiendan sus afirmaciones a partir de lo concreto

- que cuando el estudiante pregunte no den respuestas sino que utilicen el “efecto bumerang” con preguntas como ¿tú qué crees? ¿quién tiene alguna idea? ¿de qué otra manera se puede explicar?

- que aprovechen los errores (propios y de los alumnos) para encontrar la causa y corregirlos (es más importante y útil el proceso de corrección que el no cometer errores).

Una de las mejores formas de evaluar si se logró el objetivo de la clase es que soliciten a sus alumnos que inventen algún ejercicio, problema o disfraz de lo que se vio en clase, que lo hagan mentalmente y algunos lo digan en voz alta. Cuando hacen estos inventos, nos están demostrando que ya se apropiaron del conocimiento. La utilización del lenguaje simbólico escrito (números, signos y su acomodo), refleja los procesos mentales y constituye el cierre del proceso de aprendizaje de cada sesión. 
Estructura de la clase.

\section{- Introducción del tema/Activación de conocimiento.}

\section{Etapa concreta.}

Escribe en el tablero el tema Su meta de la clase . Utiliza organizadores previos discursivas redes semánticas ilustraciones _ analogías pistas

Por medio de materiales o conceptos conocidos según el tema (regletas, geoplano, recortes de periódicos, ejemplos conocidos por los estudiantes, orientaciones dirigidas y preguntas, graficación) entusiasma a los alumnos y aprovecha lo que ellos hacen para destacar lo más notable del tema__ incentiva a los más tímidos y refuerza la seguridad y confianza en sí mismos . Motiva Favorece la creatividad de los alumnos

Como estrategia para promover el aprendizaje autónomo preguntó a sus alumnos si cada uno escribió sus metas e hizo que al menos dos la leyeran

\section{- Nueva información/ estrategias de enseñanza.}

\section{Etapa semiconcreta.}

Lograda la relación matemática aprendida con el nuevo conocimiento que desea introducir (incluye la verbalización de los alumnos y la socialización) se acuden a estrategias de aprendizaje y enseñanza dependiendo del contenido matemático con la finalidad de hacer más eficiente el proceso de aprendizaje. (Sáenz, Lebrija, 2014). Se van integrando los elementos (geoplano o regletas, pizarrón y libro) para lograr un aprendizaje sólido. Manejó materiales y libro . Indique las estrategias utilizadas: toma de apuntes comprensión lectora resolución de problemas Pueden también ser utilizadas composición escrita resumen cuestionario, otras . El ambiente que se genera en los grupos es de trabajo y participación en las actividades _ Favorece que los alumnos hablen sobre lo que están haciendo_. Todos trabajan con entusiasmo y tranquilidad_. Se establece un clima relajado y espontáneo El profesor logra el control del grupo . Señale cómo favoreció el enfoque constructivista 1, 2, 3, 4, otro.

\section{- $\quad$ Cómo evaluó / cierre}

Señale que estrategias educativo sociales de organización utilizó: tarea: agenda; de información: cuadro sinóptico, mapa conceptual, mapa mental; de información matemática:, algoritmos, nemotécnicas, prueba corta, pasar al tablero.

Señale qué estrategias que fomentan la relación alumno - maestro utilizó: instrucción directa, aprendizaje por indagación, preguntas, simulación. 
Señale que estrategias de estudio solicitó: rompecabezas, aprendizaje cooperativo, aprendizaje en equipo. (STAD, TGT, TAI, CIRC), aprendiendo juntos, investigación en grupo, cooperación guiada.

\begin{tabular}{|c|c|}
\hline COMPETENCIAS QUE SE PROMOVIERON & \\
\hline Actitudes positivas y constructivas & \\
\hline Valores & \\
\hline $\begin{array}{c}\text { HABILIDADES / DESTREZAS Las habilidades del pensamiento lógico } \\
\text { promueven el desarrollo educativo del estudiante. Señale cuál(es) se promovieron }\end{array}$ & \\
\hline $\begin{array}{c}\text { Reversibilidad: al hacer las operaciones hacia delante y hacia atrás, al plantear que } \\
\text { descubran los elementos de una figura dada. (área y perímetro) y después pedir que } \\
\text { construyan la figura a partir de sus elementos. }\end{array}$ & \\
\hline $\begin{array}{c}\text { Flexibilidad de pensamiento: se favorece al buscar otras opciones para poder llegar } \\
\text { al mismo resultado con preguntas como: ¿quién lo hizo de otra manera? ¿a quién se } \\
\text { le ocurre otra forma de resolverlo? }\end{array}$ & \\
\hline $\begin{array}{c}\text { Construcción del conocimiento: mediante la creación de situaciones para que los } \\
\text { alumnos descubran los conceptos y las relaciones, confrontándolos para que } \\
\text { expresen su idea con sus propias palabras. }\end{array}$ & \\
\hline $\begin{array}{c}\text { Creatividad: pedir a los estudiantes que inventen otras aplicaciones del concepto o } \\
\text { procedimiento que se está aprendiendo, a través de disfraces, ejercicios o problemas. }\end{array}$ & \\
\hline $\begin{array}{c}\text { Aplicación a casos reales: cuando se promueve la aplicación de los conceptos a } \\
\text { situaciones que forman parte de la realidad de los niños; es una de las mejores } \\
\text { formas de llevar a cabo la “memoria generalizada”: aplicación de relaciones } \\
\text { similares a situaciones diferentes. }\end{array}$ & \\
\hline $\begin{array}{c}\text { Lenguaje algebraico: el uso de los símbolos y estructuras algebraicas (ecuaciones) } \\
\text { refleja el paso hacia el nivel de "pensamiento formal” (la abstracción) cuando el } \\
\text { niño lo maneja de manera personal. }\end{array}$ & \\
\hline
\end{tabular}

\section{Siguió la planeación \\ Comentarios y sugerencias}

Firma de la asesora

Firma del educador 